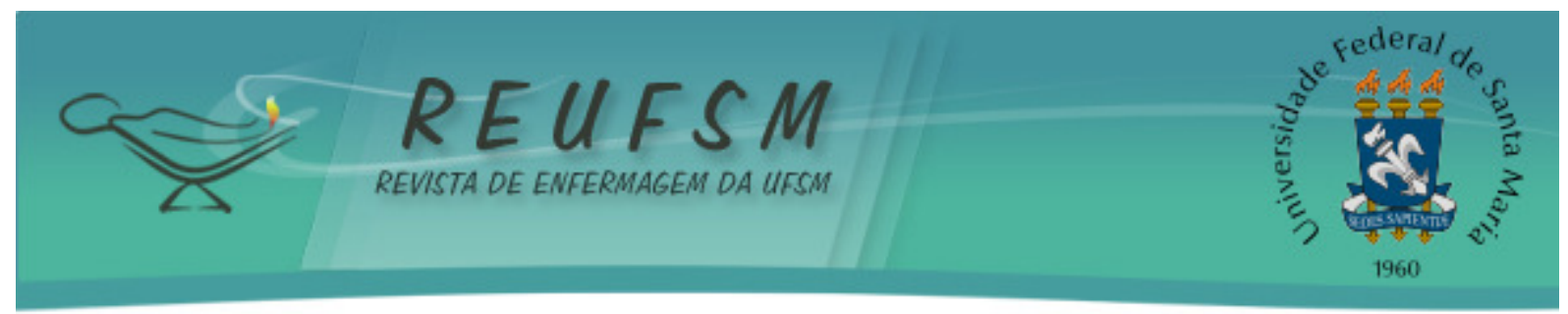

ARTIGO ORGINAL

\title{
ADOECIMENTO E USO DE MEDICAMENTOS PSICOATIVOS ENTRE TRABALHADORES DE ENFERMAGEM DE UNIDADES DE TERAPIA INTENSIVA
}

\section{ILLNESS AND THE USE OF PSYCHOACTIVE DRUGS AMONG NURSING WORKERS AT INTENSIVE CARE UNITS}

\section{LA ENFERMEDAD Y EL USO DE MEDICAMENTOS PSICOACTIVOS ENTRE LOS TRABAJADORES DE ENFERMERÍA DE UNIDADES DE CUIDADOS INTENSIVOS}

\author{
Tainara Genro Vieira ${ }^{1}$ \\ Carmem Lúcia Colomé Beck ${ }^{2}$ \\ Caliandra Marta Dissen ${ }^{3}$ \\ Silviamar Camponogara ${ }^{4}$ \\ Mariangela Gobatto ${ }^{5}$ \\ Alexa Pupiara Flores Coelho ${ }^{6}$
}

RESUMO: Objetivo: identificar o adoecimento e uso de medicamentos psicoativos entre trabalhadores de enfermagem de Unidades de Terapia Intensiva. Método: estudo descritivo com abordagem quantitativa, realizado em Unidades de Terapia Intensiva Pediátrica, Neonatal e Adulto de um hospital de ensino no segundo semestre de 2011, com participação de 49 trabalhadores de enfermagem. O instrumento foi um questionário autoaplicável, com análise por meio de estatística descritiva simples. Resultados: $28,5 \%$ dos trabalhadores usam medicação psicoativa; $24,4 \%$ destes referem que os medicamentos foram prescritos. $24,4 \%$ dos sujeitos tem alguma doença psíquica, sendo a mais citada a depressão com 14,2\%. O turno em que mais se evidenciou trabalhadores com doença psíquica foi o noturno com 43,7\%. Ainda, $63,2 \%$ dos trabalhadores referem que se automedicam, utilizando drogas antidepressivas com 12,2\%. Conclusões: é necessário criar estratégias que evitem o adoecimento, o uso de medicamentos psicoativos e a automedicação por parte dos trabalhadores de saúde.

Descritores: Enfermagem; Saúde do trabalhador; Unidades de terapia intensiva; Psicotrópicos.

ABSTRACT: Aim: to identify the disease and use of psychoactive drugs among nursing staff of the Intensive Care Unit. Method: A descriptive study with a quantitative approach, performed in the Pediatric Intensive Care Units, Neonatal and Adult of a teaching hospital in the second half of 2011, with the participation of 49 nursing staff. The instrument was self-administered questionnaire with analysis by simple descriptive statistics. Results: $28.5 \%$ of employees use psychoactive medication, $24.4 \%$ of these reported that the drugs were prescribed. $24.4 \%$ of the subjects have any mental illness, the most cited depression

\footnotetext{
${ }^{1}$ Enfermeira. Especializanda em Terapia Intensiva com ênfase em Oncologia e Controle de Infecção Hospitalar pelo Centro Universitário Franciscano (UNIFRA/RS). Enfermeira assistencial no Hospital de Caridade e Beneficência de Cachoeira do Sul, RS, Brasil. E-mail: tatigenro@hotmail.com

${ }^{2}$ Enfermeira. Doutora em Enfermagem. Docente do Departamento de Enfermagem da Universidade Federal de Santa Maria (UFSM). Santa Maria, RS, Brasil. E-mail: carmembeck@gmail.com

${ }^{3}$ Enfermeira. Enfermeira assistencial no Hospital de Caridade de Três Passos, RS, Brasil. E-mail: kalidissen@yahoo.com.br

${ }^{4}$ Enfermeira. Doutora em Enfermagem. Docente do Departamento de Enfermagem da UFSM. Santa Maria, RS, Brasil. E-mail: silviaufsm@yahoo.com.br

${ }^{5}$ Enfermeira. Mestre em Enfermagem pela UFSM. Especialista em Enfermagem do Trabalho pela Universidade do Contestado (UnC/SC). Docente do Instituto Federal de Educação, Ciência e Tecnologia do Paraná, campus de Palmas, PR, Brasil. E-mail: mariangela.gobatto@ifpr.edu.br

${ }^{6}$ Acadêmica do Curso de Graduação em Enfermagem da UFSM. Santa Maria, RS, Brasil. E-mail: alexa.p.coelho@hotmail.com
} 


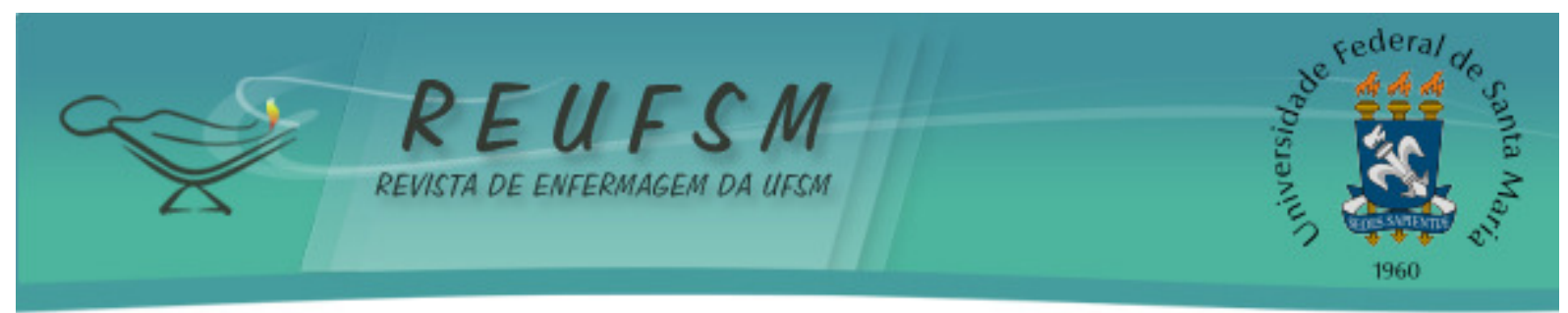

with $14.2 \%$. The shift was evident that most workers with mental illness was the night with 43.7\%. Still, $63.2 \%$ of workers reported that self-treatment using antidepressant drugs with $12.2 \%$. Conclusion: it is necessary to create strategies that avoid the illness, the use of psychoactive drugs and self-medication by health workers.

Descriptors: Nursing; Occupational health; Intensive care units; Psychotropic drugs.

RESUMEM: Objetivo: identificar la enfermedad y el uso de medicamentos psicoactivos en los trabajadores de enfermería de Unidad de Cuidados Intensivos. Método: Estudio descriptivo, abordaje cuantitativo, realizado en las Unidades Pediátricas, Neonatales y Adultas de un hospital universitario en 2011, con la participación de 49 profesionales de enfermería. El instrumento fue un cuestionario auto aplicable con el análisis de estadísticas descriptivas simples. Resultados: El $28,5 \%$ de los trabajadores utilizan medicamentos psicoactivos, el $24,4 \%$ informaron que los medicamentos fueron prescritos, el $24,4 \%$ tienen alguna enfermedad mental, la depresión es la más citada con el 14,2\%. El turno que más se evidenció trabajadores con enfermedad mental fue el nocturno con el 43,7\%. Sin embargo, el 63,2\% de los trabajadores informó que el auto tratamiento, con fármacos antidepresivos con 12,2\%. Conclusión: Es necesario crear estrategias que eviten la enfermedad, el uso de drogas psicoactivas y la automedicación por los trabajadores de salud.

Descriptores: Enfermería; Salud laboral; Unidades de cuidados intensivos; Psicotrópicos.

\section{INTRODUÇÃO}

$\mathrm{Na}$ atualidade, as transformações do mundo do trabalho têm apontado para a necessidade de investigação e tratamento do sofrimento e do desconforto físico e psíquico apresentado pelos trabalhadores, em especial, os da saúde. ${ }^{1}$ Nesse sentido, o trabalho da enfermagem pode apresentar alguns elementos capazes de comprometer o processo de viver saudável dos seus trabalhadores, tornando-os mais suscetíveis à depressão e ao cansaço. ${ }^{2}$

Especificamente no ambiente das Unidades de Terapia Intensiva (UTI's), o processo de desgaste físico e psíquico advindo da sobrecarga de trabalho pode ser gerador de estresse, prejudicando as condições de trabalho e as relações organizacionais. ${ }^{3}$ Além disso, a gravidade e complexidade do estado clínico dos pacientes, somadas a alta taxa de mortalidade que, em estudo realizado em uma UTI Adulto de um hospital universitário atingiu $50 \%,{ }^{4}$ podem mobilizar instabilidade psicoemocional e sofrimento do trabalhador de enfermagem.

Por isso, para obter conforto diante de perturbações de ordem física ou psíquica, esses trabalhadores recorrem ao uso de terapias medicamentosas, principalmente utilizando-se da automedicação. ${ }^{2}$

Em particular, o consumo de substâncias psicoativas faz parte do cotidiano dos trabalhadores de enfermagem. ${ }^{5}$ Segundo o Observatório Brasileiro de Informações Sobre Drogas $(\mathrm{OBID})^{6}$, os medicamentos psicoativos (também denominados psicotrópicos) são substâncias capazes de atuar no Sistema Nervoso Central, deprimindo-o (a exemplo dos álcoois, barbitúricos, benzodiazepínicos e opiáceos), estimulando-o (como as anfetaminas, a cocaína e o tabaco) ou perturbando-o (como a maconha, alucinógenos, anticolinérgicos, etc). Além do uso terapêutico, essas substâncias costumam ser utilizadas para o aumento da sensação de bem estar, sem prescrição médica. ${ }^{7}$

Diante do exposto, deve-se atentar para os efeitos resultantes do uso dessas substâncias que podem envolver alterações na mente, no corpo e na conduta, o que traz riscos tanto para as pessoas que as utilizam, quanto para os que com elas convivem. ${ }^{2}$ Diante disso, a realização de estudos relacionados ao uso de drogas psicoativas por profissionais de enfermagem intensivistas é relevante, considerando que esta questão interfere, diretamente, na saúde e qualidade de vida dos trabalhadores. Além disso, não pode ser desconsiderado o 


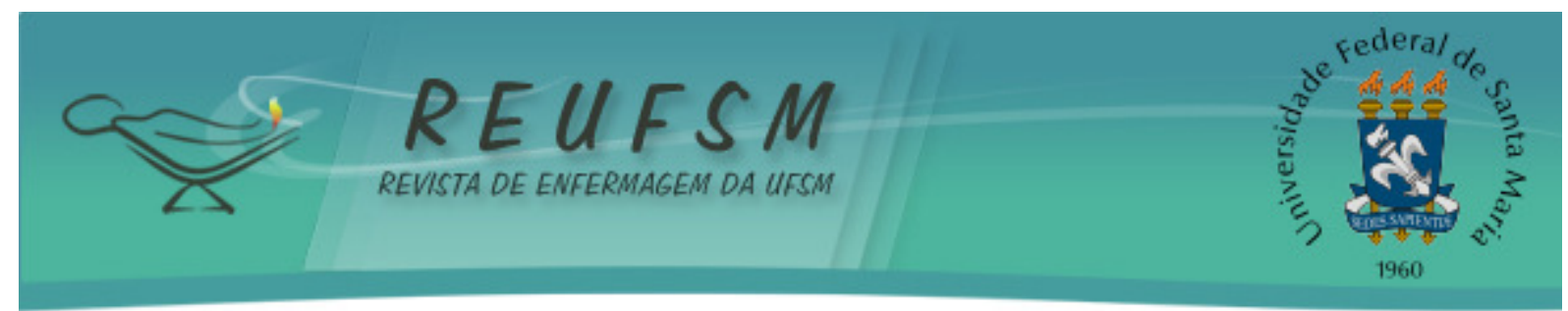

fato de que, o uso de algumas medicações psicoativas, por parte dos trabalhadores de enfermagem, pode interferir no processo assistencial, especialmente em UTI'S.

Face a relevância do tema, desenvolveu-se uma investigação sobre adoecimento e uso de substancias psicoativas entre trabalhadores de enfermagem de unidades de terapia intensiva. Neste estudo foi considerado o uso de medicamentos psicoativos prescritos ou não pelo profissional médico, sendo destacados os medicamentos antidepressivos, estimulantes, antipsicóticos, ansiolíticos/benzodiazepínicos, depressores, alucinógenos, hipnóticos e analgésicos.

Tendo em vista estas considerações, estabeleceu-se como questão de pesquisa: existe adoecimento e/ou uso de medicamentos psicoativos entre trabalhadores de enfermagem de UTI's? E como objetivo, identificar aspectos relacionados ao adoecimento e uso de medicamentos psicoativos entre trabalhadores de enfermagem de UTI's.

\section{MÉTODO}

Trata-se de um estudo do tipo descritivo, com abordagem quantitativa. ${ }^{8} 0$ cenário do estudo se constituiu das Unidades de UTI's Pediátrica, Neonatal e Adulto de um hospital de ensino localizado no Rio Grande do Sul, Brasil. A coleta dos dados foi realizada no período de outubro a novembro de 2011.

Os sujeitos da pesquisa foram os trabalhadores da equipe de enfermagem (auxiliares, técnicos em enfermagem e enfermeiros) das referidas UTI's, totalizando 108. Assim, definiu-se como critério de inclusão: ser trabalhador de enfermagem atuante nas UTI's Neonatal, Pediátrica ou Adulto e como critérios de exclusão, trabalhadores terceirizados atuantes nestas unidades tendo em vista a grande variabilidade quanto ao tempo de atuação no hospital, bem como sua rotatividade nos diferentes setores da instituição e aqueles que no período da coleta de dados encontravam-se afastados por motivos de férias, licença maternidade, adoecimento, etc.

Atenderam aos critérios de inclusão do estudo 75 trabalhadores da equipe de enfermagem, sendo que foi possível envolver 59 trabalhadores. Desses, 49 devolveram o questionário respondido, os quais se constituíram em sujeitos do estudo.

Como instrumento de coleta dos dados utilizou-se um questionário autoaplicável com questões fechadas relacionadas aos dados sociodemográficos e questões abertas relacionadas com o objetivo do estudo. Os questionários foram entregues em um envelope fechado individualmente nas UTI's durante o turno de trabalho, priorizando o momento de chegada e de saída do trabalhador. No momento da entrega foi acordado com o trabalhador o prazo de devolução do questionário sendo, em geral, de uma semana após o recebimento do mesmo.

A identidade dos trabalhadores foi mantida em sigilo e foi fornecida a eles uma cópia do Termo de Consentimento Livre e Esclarecido. Destaca-se que durante todas as etapas da realização do estudo, foram atendidas as Diretrizes e Normas da Resolução $N^{\circ}$ 196/96 do Conselho Nacional de Saúde. ${ }^{9}$ Além disto, previamente à realização do mesmo, obteve-se a autorização da instituição onde os dados foram coletados, bem como a aprovação do Comitê de Ética em Pesquisa com Seres Humanos do Protocolo do Projeto de Pesquisa, conforme o Parecer № 0197.0.243.000-11.

Para análise dos dados foi utilizada a estatística descritiva simples ${ }^{8}$, pois estas apresentavam questões diretas cujas respostas se limitavam a informações objetivas. Assim, foi possível quantificar, em forma de porcentagem, os dados obtidos.

As questões fechadas contemplavam aspectos sociodemográficos e laborais dos trabalhadores, enquanto que as abertas versavam sobre doenças em geral; doenças 


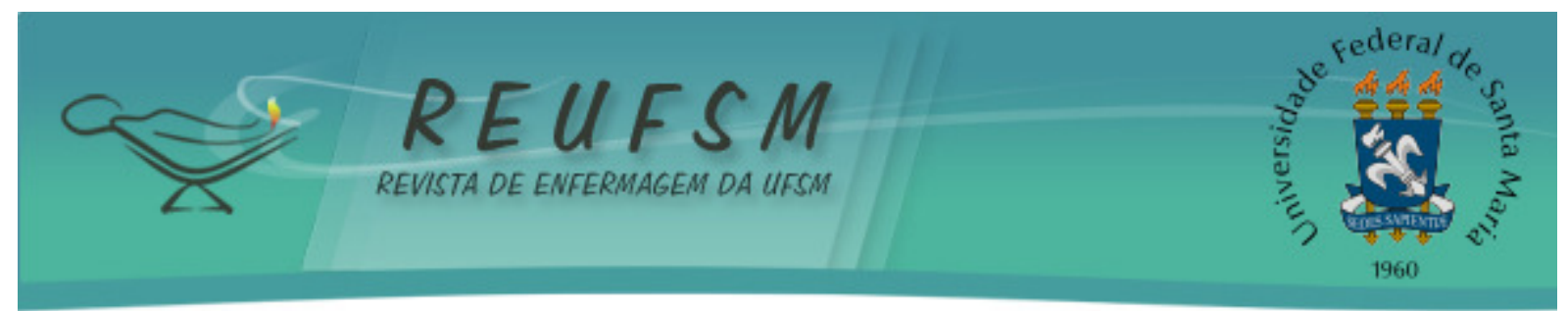

psicológicas diagnosticadas pelo médico; uso de medicamentos contínuos e psicoativos prescritos pelo médico e a automedicação.

\section{RESULTADOS}

Em relação as variáveis sociodemográficas e laborais dos sujeitos, observou-se idade média de 42 anos, com variação mínima de 22 anos e máxima de 66 anos de idade. Em relação ao sexo, 93,8\% $(n=46)$ eram mulheres e $6,2 \%(n=3)$ eram homens, sendo que $71,4 \%(n=35)$ eram casados e $77,5 \%(n=38)$ tinham filhos.

No que se refere a categoria profissional, $30,6 \%(n=15)$ eram enfermeiros, $38,7 \%$ $(n=19)$ eram técnicos em enfermagem e $30,6 \%(n=15)$ eram auxiliares de enfermagem. Em relação ao local de trabalho, 40,8\% $(n=20)$ exerciam suas atividades na UTI neonatal; $20,4 \%$ $(n=10)$ na UTI pediátrica e $38,7 \%(n=19)$ na UTI adulto. Destaca-se que, $14,2 \%$ dos trabalhadores referiram possuir mais de um emprego.

Em relação ao turno de trabalho, $18,3 \%(n=9)$ exerciam suas atividades no turno da manhã; $24,4 \%(n=12)$ no turno da tarde; $53 \%(n=26)$, no turno da noite e $10,4 \% \quad(n=5)$ informaram trabalhar em turnos mistos. Em relação ao tempo de serviço na função de auxiliar, técnico ou enfermeiro, a média de tempo de serviço na enfermagem foi de 17,6 anos. Já no que se refere ao trabalho em terapia intensiva, a média foi de 15 anos e na mesma unidade (Adulto, Pediátrica, Neonatal) a média foi de 12,8 anos.

Em relação as doenças em geral, os trabalhadores de enfermagem fizeram referência a 14 tipos diferentes de enfermidades. Do total de 23 trabalhadores que apresentavam alguma doença diagnosticada $(46,9 \%)$, oito $(16,3 \%)$ referiram hipotireoidismo. A segunda doença mais citada foi a Hipertensão Arterial Sistêmica (HAS) por sete sujeitos $(14,2 \%)$ e a terceira doença prevalente entre os trabalhadores da equipe de enfermagem das UTI's foi a depressão, sendo elencada por cinco $(10,2 \%)$ trabalhadores.

Outras situações patológicas mencionadas foram hiperinsulinemia por quatro trabalhadores $(8,1 \%)$ e diabetes mellitus por dois dos trabalhadores pesquisados $(4 \%)$. Não obstante, nove trabalhadores $(18,3 \%)$ mencionaram cada um deles, outras doenças ou sinais e sintomas como: estresse, ansiedade, enxaqueca, insônia, cardiopatia, endometriose, hérnia de disco, varizes e glaucoma.

Em relação as doenças psíquicas ou psiquiátricas diagnosticas por médico, estas foram referidas por 12 trabalhadores $(24,4 \%)$. Em relação ao tipo de doença que acomete a saúde psíquica dos sujeitos pesquisados, obteve-se como resposta a depressão, sendo mencionada por sete deles (14,2\%); ansiedade teve duas referências (4\%) e um trabalhador fez referência ao estresse $(2 \%)$. Dois trabalhadores $(4 \%)$ não especificaram qual doença psíquica ou psiquiátrica apresentavam. Neste estudo, doença mental psíquica ou psiquiátrica pode ser entendida como uma variação mórbida do normal, variação esta capaz de produzir prejuízo na performance global da pessoa (social, ocupacional, familiar e pessoal) e/ou das pessoas com quem convive. ${ }^{10}$

Em relação ao turno de trabalho com maior número de trabalhadores de enfermagem com doenças psíquicas ou psiquiátricas, o noturno se destacou com $35,7 \%$. Trabalhadores que alternam suas atividades nos turnos manhã, tarde e noite, ocuparam o segundo lugar nesse ranking, com três referências no que se refere as doenças psicológicas $(21,4 \%)$.

Em relação ao uso de medicação psicoativa, o mesmo foi confirmado por 14 trabalhadores de enfermagem $(28,5 \%)$, sendo que a medicação mencionada com maior frequência foram os antidepressivos, citados por seis trabalhadores $(12,2 \%)$. Os benzodiazepínicos foram mencionados por três sujeitos $(6,1 \%)$ e o uso de analgésicos teve duas menções $(4 \%)$, sendo que três trabalhadores $(6,1 \%)$ não responderam a essa questão. 


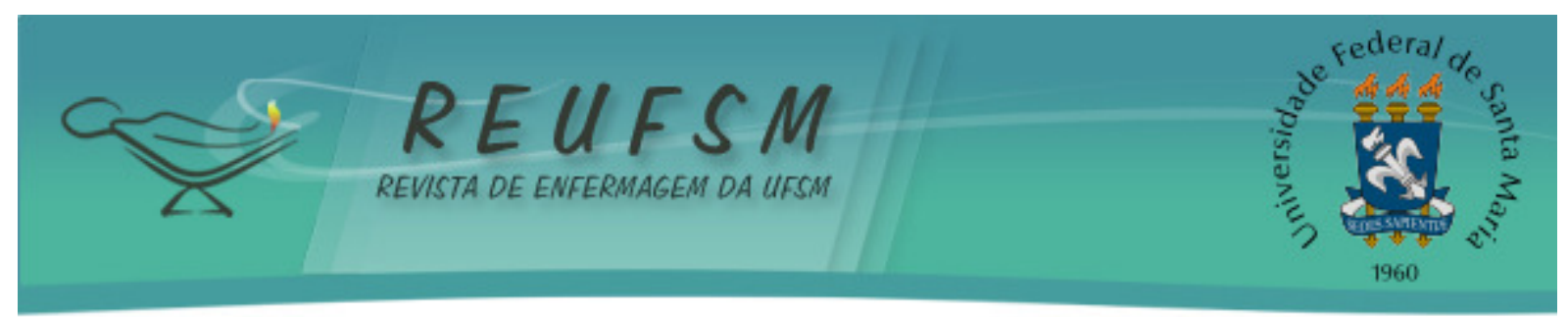

Quanto ao tempo de uso desse tipo de medicamento, a média foi de quatro anos. Em relação à frequência de consumo, nove trabalhadores (18,3\%) assinalaram o uso de psicoativo por mais de 20 dias no mês. No caso de 12 trabalhadores (24,4\%), a medicação foi prescrita pelo profissional médico; sendo que dois trabalhadores (4\%) não responderam a pergunta.

Para justificar o uso destes medicamentos, os trabalhadores de enfermagem relacionaram o estresse causado pela agitação do dia-a-dia, o cansaço, as questões referentes ao trabalho, a perda de familiares, bem como os problemas envolvendo o desempenho escolar dos filhos. Os sentimentos relatados diante deste contexto foram de tristeza, ansiedade, depressão e de não sentir-se bem sem a medicação.

Ao responderem a pergunta sobre a automedicação em relação a qualquer tipo de medicamento, 31 trabalhadores $(63,2 \%)$ responderam afirmativamente, 15 informaram não realizá-la $(30,6 \%)$ e três $(6,1 \%)$ não responderam a este questionamento.

Os analgésicos foram citados 26 vezes, os antiinflamatórios seis vezes, os antibióticos três vezes, os relaxantes musculares e os antitérmicos tiveram duas referências cada um, sendo que os antigripais, antieméticos e ansiolíticos foram mencionados uma vez cada um.

\section{DISCUSSÃO}

Dentre as doenças referidas pelos trabalhadores de enfermagem, prevaleceram o hipotireoidismo, a HAS e a depressão.

O hipotireoidismo se caracteriza por um conjunto de sinais e sintomas decorrentes da diminuição da produção dos hormônios da tireoide; tem maior incidência em mulheres e pode provocar cansaço, depressão, raciocínio lento, fraqueza muscular, pequeno ganho de peso, edema nas extremidades, dentre outros sinais e sintomas. ${ }^{11}$ Observando os sintomas da doença e o fato de serem as mulheres as mais frequentemente afetadas, afirma-se que essa doença pode interferir no ritmo de trabalho das mulheres trabalhadoras de enfermagem em UTI, uma vez que pode prejudicar sua rotina, especialmente por causar alterações no estado psicológico podendo, inclusive, ser uma das causas da depressão.

Já a HAS vem sendo considerada um problema grave de saúde pública no Brasil e no mundo, sendo um importante fator de risco para doenças cerebrovasculares, cardiovasculares e renais. ${ }^{12}$ Estudos apontam para a relação de causa e efeito entre a HAS e o estresse mental. Logo, destaca-se a necessidade de mudanças nas condutas individuais, bem como no ambiente de trabalho, tornando-o mais agradável, considerando que isso possa influenciar beneficamente na variação da pressão arterial dos trabalhadores. ${ }^{13}$

Especificamente em relação a depressão, trata-se de um transtorno que altera o humor, afetando o modo de ver o mundo, bem como de sentir a realidade. Seus sintomas podem incluir tristeza intensa, insegurança, isolamento social e familiar, apatia, desmotivação ocasionada pela perda de interesse e prazer por elementos outrora apreciados, com o agravante de que podem também ocorrer perda de memória, do apetite e da concentração, além de insônia. ${ }^{14}$

Sabe-se também que os transtornos psíquicos e do comportamento ocupam o terceiro lugar em número de auxílios-doença concedidos pelo Instituto Nacional do Seguro Social (INSS), sendo que a metade destes benefícios é concedida, por exemplo, a pessoas com depressão. ${ }^{15}$ Nesse sentido, torna-se relevante combater os fatores motivadores dessa doença, especialmente entre os trabalhadores de enfermagem, cujo trabalho demanda saúde física e, especialmente a psíquica, para o exercício efetivo do cuidado.

Estudo realizado com trabalhadores de enfermagem evidenciou fatores que podem desencadear a depressão, como: os elementos internos ao ambiente; o processo de trabalho relacionado aos turnos laborais e aos problemas de escala de serviço; à 


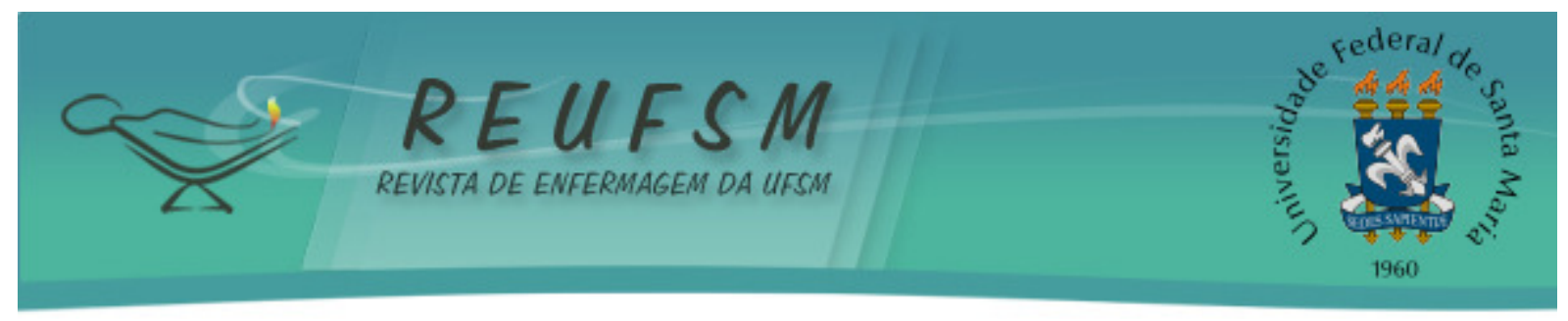

assistência aos pacientes com ênfase para a insegurança e os conflitos de interesses. Além disso, podem ser citados fatores externos, como: sexo, idade, suporte e renda familiar, trabalho doméstico e as características individuais dos sujeitos. ${ }^{16}$ A depressão causa prejuízo para a saúde do trabalhador de enfermagem e empresa empregadora, o que reitera e torna imperativa a implementação de programas de atenção à sua saúde.

A ansiedade, segunda doença de ordem psíquica mais citada pelos trabalhadores de enfermagem, é um estado psíquico-emocional que pode acarretar em perturbações fisiológicas (como dores, mudanças no apetite, palpitações, hipertensão), emocionais (como depressão, frustração, irritabilidade, desânimo), de relacionamento (como isolamento) e espirituais (sensações de vazio, martírio e apatia), podendo, ainda, assumir caráter incapacitante quando em níveis elevados. ${ }^{17}$ Este desequilíbrio pode ocorrer em virtude da pressão e do estresse oriundos do ambiente de trabalho, onde o trabalhador fica obcecado pela eficácia para ser aceito e não perder o emprego.

No que se refere ao estresse, em estudo realizado com enfermeiros trabalhadores em terapia intensiva foram constatados, como fatores estressantes: a sobrecarga de trabalho seguida de conflito de funções; desvalorização profissional; condições de trabalho inadequadas; dupla jornada de trabalho; falta de autonomia; insatisfação com o trabalho; relacionamento interpessoal frágil; baixa remuneração; presença de ruídos; ocorrência de acidentes biológicos e a ocorrência de morte de pacientes. ${ }^{18}$

Logo, percebe-se que o estresse e a ansiedade podem ser evitados ou amenizados, a partir de modificações no processo e no ambiente laborativo, as quais possibilitem que os trabalhadores sintam-se mais tranquilos, seguros, confortáveis e com maior poder de decisão. Assim, acredita-se que o trabalho dar-se-á de modo mais satisfatório o que, consequentemente, poderia favorecer o seu reconhecimento perante os pacientes, seus familiares e integrantes da equipe de saúde.

A falta de reconhecimento soma-se a elevada cobrança a qual estão submetidos esses trabalhadores, tanto em relação ao concorrido mercado de trabalho, quanto à responsabilidade exigida nos cargos que ocupam nas UTI's. Estas situações podem contribuir com o surgimento do estresse, assim como da depressão.

Defende-se que a dinâmica do reconhecimento se relaciona com o surgimento de um processo de reapropriação que extrapola o processo de resistir ao sofrimento e ao adoecimento, ao possibilitar, aos trabalhadores, a construção de saúde. Logo, para que o trabalho opere como fonte de saúde há a necessidade do reconhecimento daquele que trabalha, uma vez que no reconhecimento reside a possibilidade de dar sentido ao sofrimento vivenciado pelos trabalhadores. ${ }^{19}$

Com relação ao turno de trabalho, percebe-se, neste estudo, que os trabalhadores noturnos, bem como aqueles que não têm uma rotina fixa de trabalho e de vida, estão mais predispostos a distúrbios psíquicos. 0 trabalho noturno obriga os trabalhadores a inverterem seu padrão normal de sono e quando há uma excessiva e persistente privação do mesmo, isso pode gerar sintomas como irritabilidade, diminuição do nível de alerta, fadiga, dentre outros. ${ }^{20}$ Se ocorrerem com frequência e por tempo prolongado, esses sintomas podem resultar no desgaste físico e psíquico do trabalhador, culminando no adoecimento laboral.

Esses dados se associam aos apresentados ao estudo que aponta o trabalho noturno com um dos principais fatores para a depressão, no qual $28,4 \%$ da população de estudo obtiveram escores que sugeriam depressão. ${ }^{21}$

Ainda, considera-se relevante discutir o tempo médio de trabalho dos trabalhadores de enfermagem em UTI que referiram padecer de doenças psicológicas. Neste estudo, constatou-se que o tempo médio de atuação como trabalhador da equipe de enfermagem foi de 17,6 anos, sendo que a média do tempo de trabalho na UTI foi de 15 anos e na mesma UTI de 12,8 anos. 


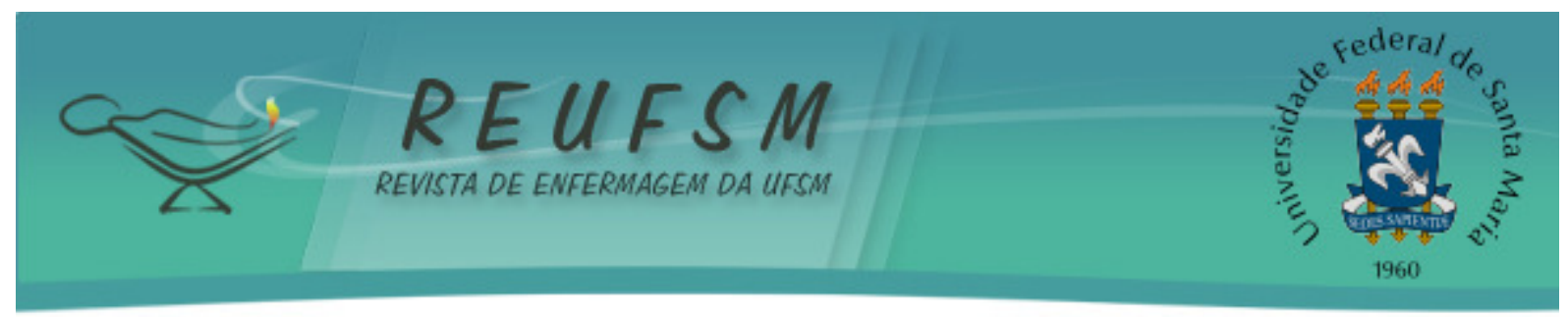

O uso de medicamentos psicoativos foi referido por considerável parcela dos trabalhadores de enfermagem, sendo que os antidepressivos, benzodiazepínicos e analgésicos foram as categorias citadas. Os benzodiazepínicos, que figuram como segundo tipo de medicação mais consumida pela população investigada, também são conhecidos como ansiolíticos. Esta classe de medicamentos estimula mecanismos no cérebro que, normalmente, equilibram estados de tensão e ansiedade, além de inibir os mecanismos que funcionam em excesso devido as tensões do dia-a-dia, resultando num estado de ansiedade. Em resposta a esta inibição, a pessoa apresenta maior tranquilidade e fica menos responsiva a estímulos externos. Como consequência desta ação, os ansiolíticos produzem uma depressão da atividade do cérebro que se caracteriza por diminuição de ansiedade, indução de sono; relaxamento muscular; redução do estado de alerta. ${ }^{22}$

Já a referência ao uso de analgésicos remete à vivência da dor pelo trabalhador de enfermagem de UTI. A incidência da dor pode estar relacionada ao processo de trabalho em unidades críticas caracterizada pelo desgaste físico, pelo alto ritmo e cargas de trabalho exaustiva para os trabalhadores, podendo acarretar no surgimento da ansiedade e do estresse. A dor, quando tratada de maneira inadequada, pode provocar efeitos danosos estando associada, por exemplo, a alterações no padrão do sono. ${ }^{17}$ Esta possível relação entre a vivência da dor, o uso de analgésicos e o trabalho de enfermagem em UTI's aponta para a vulnerabilidade desse trabalhador ao sofrimento e, potencialmente, ao adoecimento laboral.

Sabe-se que os trabalhadores de enfermagem representam a maior dentre as categorias de trabalhadores inseridos nas instituições de saúde. Entretanto, não têm tido a devida atenção dos gestores, no que se refere à promoção de qualidade de vida e saúde. 0 trabalhador, no ambiente hospitalar, está exposto a cargas de trabalho como, por exemplo, as biológicas, químicas, mecânicas, físicas fisiológicas e psíquicas. A elevada exposição às cargas psíquicas pode ser geradora de problemas como desequilíbrio mental, desgaste emocional, enxaqueca e distúrbios digestivos, comprometendo a qualidade da assistência prestada aos pacientes e a própria qualidade de vida dos profissionais de enfermagem. ${ }^{23}$

Pesquisa realizada junto a trabalhadores de enfermagem de uma UTI evidenciou a inter-relação entre o uso de medicamentos psicoativos e o estresse, ansiedade e sobrecarga de trabalho. ${ }^{5}$ Estes dados vem ao encontro do presente estudo, no sentido de que o uso do medicamento psicoativo ainda que, por vezes, sem orientação médica, representa uma resposta do trabalhador ao sofrimento vivenciado no trabalho.

A automedicação foi relatada pela maioria dos trabalhadores de enfermagem. Dentre as medicações, as mais citadas foram os analgésicos e anti-inflamatórios. Estudo aponta que os profissionais de enfermagem se automedicam com base em seus conhecimentos em relação aos efeitos provocados pelos medicamentos e que os mesmos são utilizados conforme suas necessidades, visando a minimização do transtorno psíquico ou físico. ${ }^{2} \mathrm{~A}$ maior frequência pela automedicação por analgésicos e anti-inflamatórios também foi resultado de outro estudo, igualmente realizado com trabalhadores de enfermagem. ${ }^{2}$

É possível que as dores e as inflamações sofridas pelos trabalhadores de enfermagem pesquisados estejam vinculadas à presença de distúrbios musculoesqueléticos ocasionados pelas más condições de trabalho, redução do tempo de descanso, o que leva a necessidade de realizar a automedicação. ${ }^{24}$ Nessa linha, deve-se promover um ambiente de trabalho adequado, minimizando os fatores de estresse e, consequentemente, de adoecimento para os trabalhadores de enfermagem, visando promover melhoria dos sintomas que levam ao adoecimento.

Alguns profissionais possuem conhecimento sobre os efeitos das drogas disponíveis, o que pode favorecer a automedicação. As principais queixas referidas pelos profissionais de enfermagem que os motivam a automedicação são dores em geral, gripes, resfriados, dor de cabeça e febre ${ }^{25}$, evidenciando o uso de antitérmicos, analgésicos e anti- 


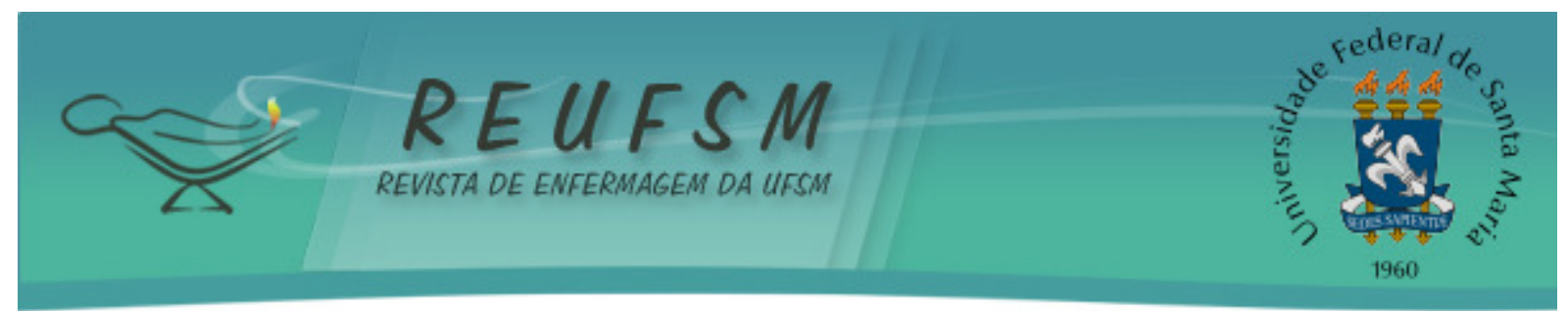

inflamatórios. Além deste grupo de medicamentos, outros estão na lista de preferência, dos trabalhadores como, por exemplo: os antiespasmódicos, antiácidos, antibióticos, anticoncepcionais, corticóides, antimicóticos, antigripais, vitaminas e benzodiazepínicos.

\section{CONSIDERAÇÕES FINAIS}

Os dados do estudo apontam para o fato de que, a medida que se considera o cotidiano do trabalho com as características próprias das unidades pesquisadas, entende-se como necessário e urgente a compreensão dos fatores de risco e de proteção para os trabalhadores de enfermagem, bem como o adoecimento e uso dessas substâncias.

Dentre os prováveis fatores de risco encontrados nesse estudo destacam-se: longo tempo de atuação exclusivamente em unidade crítica, trabalho noturno, condições de trabalho nem sempre adequadas. Outro fator que pode estar associado é a disponibilidade das substâncias no ambiente de trabalho, considerando a complexidade e gravidade dos pacientes internados nestas unidades.

Diante da problemática, acredita-se que as instituições de saúde devam ampliar a discussão acerca da relação trabalho/saúde, o que implica na avaliação dos fatores de risco aos quais os trabalhadores estão submetidos. Logo, salienta-se a necessidade de que os gestores busquem repensar a estrutura organizacional das instituições hospitalares, realizando promoção de saúde para os profissionais de enfermagem, com enfoque especial para os que atuam em UTIs.

\section{REFERÊNCIAS}

1. Martins SR, Mendes AM. Espaço coletivo de discussão: a clínica psicodinâmica do trabalho como ação de resistência. Rev Psicol Organ Trab [Internet]. 2012 maio-ago [acesso em 2013 jun 3];12(2):171-84. Disponível em: http://pepsic.bvsalud.org/pdf/rpot/v12n2/v12n2a04.pdf.

2. Baggio MA, Formaggio FM. Automedicação: desvelando o descuidado de si dos profissionais de enfermagem. Rev Enferm UERJ [Internet]. 2009 abr-jun [acesso em 2012 jul 2];17(2):224-8. Disponível em: http://www.facenf.uerj.br/v17n2/v17n2a15.pdf.

3. Fogaça MC, Carvalho WB, Martins LAN. Estudo preliminar sobre a qualidade de vida de médicos e enfermeiros intensivistas pediátricos e neonatais. Rev Esc Enferm USP [Internet]. 2010 [acesso em 2012 jul 2];44(3):708-12. Disponível em: http://www.scielo.br/pdf/reeusp/v44n3/22.pdf.

4. Favarin SS, Camponogara S. Perfil dos pacientes internados na Unidade de Terapia Intensiva Adulto de um hospital universitário. Rev Enferm UFSM [Internet]. 2012 maio-ago [acesso em 2012 dez 24];2(2):320-9. Disponível em:

http://cascavel.ufsm.br/revistas/ojs-2.2.2/index.php/reufsm/article/view/5178/3913.

5. Dias JRF, Araújo CS, Martins ERC, Clos AC, Francisco MTR, Sampaio CEP. Fatores predisponentes ao uso próprio de psicotrópicos por profissionais de enfermagem. Rev Enferm UERJ [Internet]. 2011 jul-set [acesso em 2013 jun 3];19(3):445-51. Disponível em: http://www.facenf.uerj.br/v19n3/v19n3a18.pdf.

6. Brasil. Ministério da Justiça. Observatório Brasileiro de Informações Sobre Drogas (OBID) [Internet]. 2007 [acesso em 2013 jun 2]. Disponível em: http://www.obid.senad.gov.br/portais/OBID/index.php. 


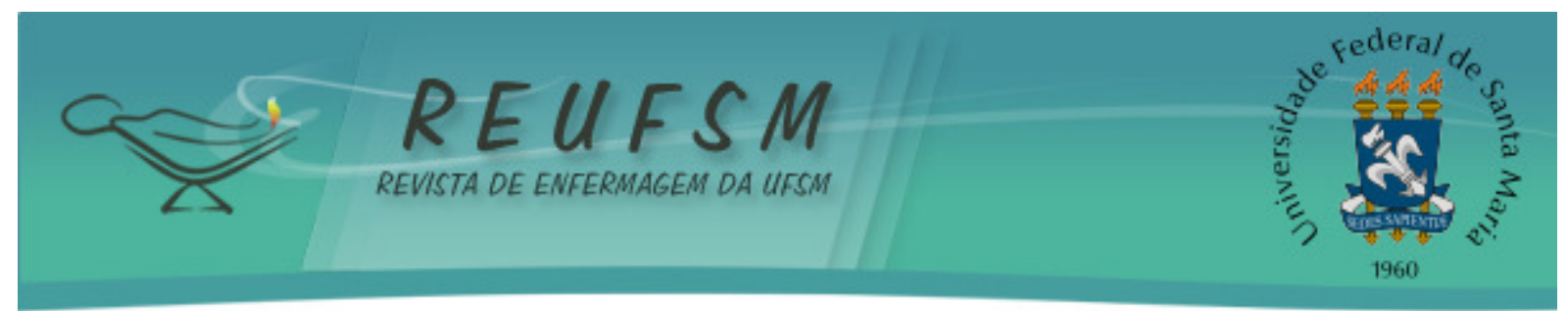

7. Nicoll RA. Introdução à farmacologia dos fármacos que agem no SNC. In: Katzung BG. Farmacologia básica e clínica. Fármacos que agem no Sistema Nervoso Central (SNC). $10^{\mathrm{a}}$ ed. Porto Alegre, RS: AMGH; 2010. 1060 p.

8. Lakatos EM, Marconi, MA. Fundamentos da metodologia científica. São Paulo (SP): Atlas; 2005.

9. Brasil. Conselho Nacional de Saúde. Resolução N 196/96, de outubro de 1996. Diretrizes e normas regulamentadoras de pesquisa em seres humanos [Internet]. 1996 [acesso em $2011 \quad$ mar 3 . Disponível http://conselho.saude.gov.br/resolucoes/reso_96.htm.

10. Ballone GJ. O que são transtornos mentais. PsiqWeb [Internet]. 2008 [acesso em 2013 jun 25].

Disponível

em: http: //www.psiqweb.med.br/site/?area=NO/LerNoticia\&idNoticia=230.

11. Czepielewski MA. Hipotireoidismo. Abc Saúde [Internet]. 2008 [acesso em 2011 out 30]. Disponível em: http://www.abcdasaude.com.br/artigo.php?248.

12. Brasil. Ministério da Saúde. Secretaria de Atenção à Saúde. Departamento de Atenção Básica. Hipertensão arterial sistêmica para o Sistema Único de Saúde [Internet]. 2006 [acesso em 2011 out 30]. Disponível em: http://bvsms.saude.gov.br/bvs/publicacoes/caderno_atencao_basica15.pdf. (Cadernos de Atenção Básica, 15).

13. Fonseca FCA, Coelho RZ, Nicolato R, Malloy-Diniz LF, Silva Filho HC. A influência de fatores emocionais sobre a hipertensão arterial. J Bras Psiquiatr [Internet]. 2009 [acesso em 2011 out 30];58(2):128-34. Disponível em: http://www.scielo.br/pdf/jbpsiq/v58n2/v58n2a11.pdf.

14. Teixeira S. A depressão no meio ambiente do trabalho e sua caracterização como doença do trabalho. Rev Trib Reg Trab [Internet]. 2007 jul-dez [acesso em 2011 nov 1];46 (76):27-44. Disponível http://www.trt3.jus.br/escola/download/revista/rev_76/Sueli_Teixeira.pdf.

15. Brasil. Ministério da Previdência Social. Informações estatísticas relativas à segurança e saúde ocupacional. Auxílios-doença acidentários e previdenciários segundo os códigos da Classificação Internacional de Doenças - CID-10 [tabela][Internet]. 2011 [acesso em 2012 jul 26]. Disponível em: http: //www. previdencia.gov.br/conteudoDinamico.php?id=502.

16. Manetti ML, Marziale MHP. Fatores associados à depressão relacionada ao trabalho de enfermagem. Estud Psicol [Internet]. 2007 [acesso em 2011 nov 15];12(1):79-85. Disponível em: http://www.scielo.br/pdf/epsic/v12n1/a10v12n1.pdf.

17. Smeltzer SC, Bare GB, Hinkle JL, Cheever KH, editores. Brunner \& Suddarth: Tratado de Enfermagem Médico-Cirúrgica. 12ª ed. Rio de Janeiro (RJ): Guanabara Koogan; 2011. V. 2.

18. Santos FD, Cunha MHF, Robazzi MLCC, Pedrão LJ, Silva LA. O estresse do enfermeiro nas unidades de terapia intensiva adulto: uma revisão da literatura. SMAD Rev Eletrônica Saúde Mental Alcool Drog [Internet]. 2010 [acesso em 2011 nov 18];6(1):1-16. Disponível em: http: //pepsic.bvsalud.org/pdf/smad/v6n1/14.pdf.

19. Mendes AMB, Vieira AP, Morrone CF. Prazer, sofrimento e saúde mental no trabalho de teleatendimento. RECADM [Internet]. 2009 [acesso em 2012 maio 8];8(2):151-8. Disponível em: http://revistas.facecla.com.br/index.php/recadm/article/view/360/456.

20. Medeiros SM, Macêdo MLAF, Oliveira JSA, Ribeiro LM. Possibilidades e limites da recuperação do sono de trabalhadores noturnos de enfermagem. Rev Gaúcha Enferm 


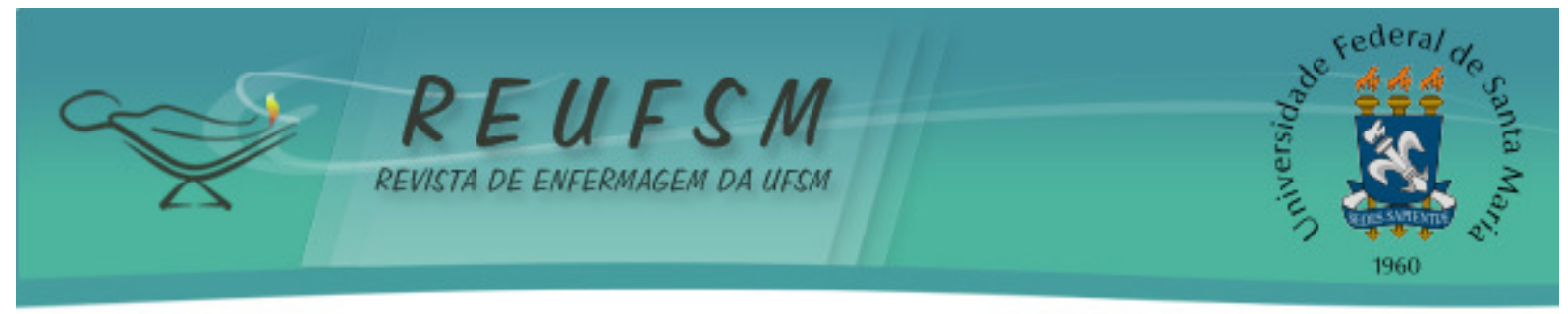

[Internet]. 2009 mar [acesso em 2011 nov 18];30(1):92-8. Disponível em: http://seer.ufrgs.br/RevistaGauchadeEnfermagem/article/download/5111/6568.

21. Vargas D, Dias APV. Depression prevalence in Intensive Care Unit nursing workers: a study at hospitals in a northwestern city of São Paulo State. Rev Latinoam Enferm [Internet]. 2011 Sept-Oct [acesso em 2013 jun 25];19(5):1114-21. Disponível em: http: //www.scielo.br/pdf/rlae/v19n5/08.pdf.

22. Ballone GJ, Ortolani IV. Psicofarmacologia para não psiquiatras, ansiolíticos. PsiqWeb [Internet]. 2005 [acesso em 2011 nov 18]. Disponível em: http://www.psiqweb.med.br.

23. Mininel VA, Babtista PCP, Felli VEA. Cargas psíquicas e processos de desgaste em trabalhadores de enfermagem de hospitais universitários brasileiros. Rev Latinoam Enferm [Internet]. 2011 mar-abr [acesso em 2011 nov 19];19(2). Disponível em: http://www.scielo.br/pdf/rlae/v19n2/pt_16.pdf.

24. Barros ARR, Griep RH, Roterberg L. Automedicação entre os trabalhadores de enfermagem de hospitais públicos. Rev Latinoam Enferm [Internet]. 2009 nov-dez [acesso em 2011 nov 19];17(6). Disponível em: http://www.scielo.br/pdf/rlae/v17n6/pt_14.pdf.

25. Paredes NP, Miasso Al, Tirapelli CR. Consumo de benzodiazepínicos sem prescrição médica entre estudantes do primeiro ano da Escola de Enfermagem da Universidade de Guayaquil, Equador. Rev Latinoam Enferm [Internet]. 2008 maio-jun [acesso em 2013 maio 25];16(Esp). Disponível em: http://www.scielo.br/pdf/rlae/v16nspe/pt_21.pdf.

Data de recebimento: $21 / 12 / 2012$

Data de aceite: 02/08/2013

Contato com autor responsável: Tainara Genro Vieira

E-mail: tatigenro@hotmail

Endereço: Rua Izidoro Grassi, $n^{\circ}$ 23, ap. 207, Bloco 02, Bairro Medianeira, Santa Maria, RS. CEP 97060-310 